\title{
Wie schaffen wir Anreize für Forschung und Entwicklung (F\&E) für Versorgungs- und Systeminnovationen in einem nach wie vor körperschaftlich geregelten Gesund- heitswesen?
}

\section{- Eine Diskussion des vorgelegten White Papers und der bisher eingegangenen Kommentare}

Versorgungsinnovationen und damit Systeminnovationen sind für die Zukunft des Gesundheitswesens, die Bewältigung des demografischen Wandels und die Vorbereitung der deutschen Gesundheitswirtschaft auf die Herausforderungen der Europäisierung von ganz entscheidender Bedeutung. Die Anschubfinanzierung der Integrierten Versorgung (IV) hat in insgesamt 5.475 Verträgen (Stand 1.Q.2008, BQS)1 wichtige Anstöße für die Entwicklung von Versorgungsinnovationen im Gesundheitswesen gegeben und hat zu großen Lerneffekten sowohl bei den Leistungserbringern wie bei den Kostenträgern geführt. Sie hat aber noch lange nicht das Potenzial ausgefüllt, das sich noch an Systemwirtschaftlichkeit für die Zukunft verbirgt. Mit dem Auslaufen der Anschubfinanzierung Ende 2008 und auch aufgrund von problematischen Anreizwirkungen des Gesundheitsfonds muss deshalb heute die Diskussion geführt werden, mit welchen Methoden eine Verstetigung der Entwicklung und Einführung von Versorgungsinnovationen befördert werden kann.

\section{H. Hildebrandt, Hamburg}

Am 3. Juni 2008 hat die Hamburger Beratungsfirma Hildebrandt GesundheitsConsult GmbH dazu ein White Paper „Anreize für Forschung und Entwicklung (F\&E) für Versorgungs- und Systeminnovationen im Gesundheitswesen “ vorgelegt und zur Diskussion gestellt ${ }^{2}$. Ihre Kernforderung ist darin die stärkere Gesamtausrichtung des Gesundheitswesens und der ökonomischen Anreizmechanismen aller Beteiligten auf eine Ergebnisorientierung, d. h. den produzierten Gesundheitsnutzen über den gesamten Behandlungsprozess und Krankheitszyklus hinweg.

Das White Paper diskutiert die Herausforderungen und die Bedarfe für eine Verstetigung von Versorgungsinnovationen in diesem Sinne und schlägt ein Maßnahmenbündel zur Entwicklung und Förderung einer Kultur von Forschung und Entwicklung (F+E) für Versorgungsinnovationen im Gesundheitswesen vor. Vier Kernelemente wurden dabei herausgehoben:

Helmut Hildebrandt, Hildebrandt GesundheitsConsult GmbH, Hamburg
Einführung eines F+E-Anschubetats als Teil des Gesundheitsfonds: Die Krankenkassen sollen für einen gewissen Anteil der Gesamtausgleichsbeträge des Gesundheitsfonds (wir denken an eine Größenordnung von 1-3\%, d.h. 1,5 bis 4,5 Milliarden Euro) einen Nachweis führen, dass sie entsprechende Investitionen in F+E für Versorgungs- und Systeminnovationen vorgenommen haben. Wenn sie diesen Nachweis nicht führen können, dann geht dieser Anteil an den Gesundheitsfonds zurück und steht allen Krankenkassen wiederum für das nächste Jahr zur Verfügung. (Überführung der bisherigen Anschubfinanzierung mit dem 1\% Einbehalt bei den Vergütungen der Krankenhäuser und Ärzteschaft jetzt auf eine entsprechende Regelung bei den Krankenkassen)

Transparenzverpflichtung der Krankenkassen zu Berichten zur Versorgungsqualität ihrer Versicherten anhand eines definierten Sets von Qualitätsindikatoren (Weiterentwicklung der bereits vorhandenen Qualitätsberichtsverpflichtungen der Krankenhäuser, jetzt auch auf Krankenkassen) 
- Ergebnisbezogene Modulation der selektiv- wie kollektivvertraglichen Vergütungen in Abhängigkeit von dem geschaffenen Gesundheitsnutzen (im angloamerikanischen Sprachraum u.a. als „Pay-for-Performance“ diskutiert).

- Gründung eines Kapitalfonds zur Investitionsfinanzierung für Unternehmensformen im Bereich von Versorgungsinnovationen (etwa durch zinsbegünstigte Darlehen analog der Mittelstandsförderung über die Bankengruppe der Kreditanstalt für Wiederaufbau)

Das White Paper greift mit seinen Vorschlägen zurück auf eine breite Diskussion, die national wie international in den Gesundheitswissenschaften wie der Gesundheitsökonomie und im Sachverständigenrat geführt wird. Verwiesen seien dazu u.a. auf die Beiträge von Krauth/ Schwartz/Perleth/Graf von Schulenburg ${ }^{3}$, von Porter/ Teisberg4, Enthoven/Tollen5 und Lüngen/Gerber/Lauterbach6.

Zusammen mit der Vorlage des White Papers erfolgte eine Diskussion, an der sich eine ganze Reihe von Gesundheitsökonomen und Vertreter aus verschiedenen Krankenkassen intensiv beteiligten ${ }^{7}$. Diese Diskussion konzentrierte sich auf den Vorschlag der Etatisierung eines F\&E-Anschubetats im Gesundheitsfonds und auf Transparenzverpflichtungen, d.h. die Forderung nach einer Gesundheitsnutzensbilanzierung durch die Krankenkassen. Auf diese Diskussion soll in diesem Beitrag eingegangen werden und gleichzeitig die Maßnahmenvorschläge des White Papers noch einmal im Zusammenhang dargestellt werden.

\section{Bedarf einer Kultur von Forschung und Entwicklung (F+E) für Versorgungsinnovationen im Gesundheitswesen}

Wenn es eine Gemeinsamkeit aller Beteiligten im deutschen Gesundheitswesen gibt, dann sicherlich diese: Die heutigen Ablaufprozesse der Patientenversorgung sind vielfach aufgrund der sektoralen ökonomischen Begrenzungen nicht zeitgemäß, verursachen Unwirtschaftlichkeiten, sind nicht patientenfreundlich und bedürfen dringend der Veränderung. Aus epidemiologischer und gesundheitsökonomischer Sicht, so u. a. in den letzten Gutachten des Sachverständigenrats, wird in diesem Zusammenhang die mangelnde Ergebnisorientierung der Versorgungsprozesse beschrieben. Die jeweiligen Verbände der Leistungserbringer beklagen die Begrenzung und Sinnwidrigkeit der sektoralen Budgetierung mit ihrer inhärenten Innovationsbremse durch die Deckelung der Budgets. Die Kostenträger sehen insbesondere die fehlende Effizienz des gesamten Behandlungsprozesses, wenn etwa aufgrund sektoraler Budgetierung ambulante Leistungserbringer auf eine eigentlich mögliche intensivierte ambulante Betreuung zugunsten einer stationären Einweisung verzichten. Die Industrie wiederum beklagt, dass die Einführung neuer Techniken und Verfahren, die positive Wirkungen in einem anderen Sektor oder bei einem anderen Kostenträger haben, durch den „Sektorblick“ der jeweiligen Leistungserbringer behindert wird und so Wettbewerbsnachteile für Industrie wie das Gesundheitssystem als Ganzem produziert.

Als zum Beginn des Jahres 2000 die $§ \S 140 a-h$ zur Integrierten Versorgung in das SGB V neu eingeführt wurden, geschah dies unter folgenden Überlegungen:

- Die bisher in Deutschland vorherrschende sektoral organisierte Gesundheitsversorgung verursacht Abstimmungsprobleme und Unwirtschaftlichkeiten.

- Die sektorale Versorgung führt notwendigerweise zu höheren Kosten für das Gesamtsystem, da jeder Sektor auf seine eigene Wirtschaftlichkeit achtet aber nicht auf die Gesamtwirtschaftlichkeit quer über den Behandlungsprozess. Aus Erfahrungen in der Schweiz und in den USA gab es Schätzungen, die bis zu 30\% Einsparmöglichkeiten prognostizierten.

- Statt der medizinischen Effizienz der Behandlung und eines gesundheitlichen Outcomes steht für die einzelnen Sektoren die je eigene Umsatz- und wirtschaftliche Erfolgsmaximierung im Mittelpunkt des Handelns - diese Orientierung erweist sich als ein entscheidendes Hindernis für die Entwicklung von Prozesseffizienz.

- Da sich das System auf der zentralen Ebene als nahezu reformunfähig erwiesen hat, sollten auf der lokalen Ebene Reformen und unterschiedliche Experimente ermöglicht werden. ${ }^{8}$

Die Anschubfinanzierung der Integrierten Versorgung mit der ihr innewohnenden Möglichkeit der Überbrückung der sektoralen Budgetierung ist im Rückblick bei aller Kritik, im Detail durchaus als ein erfolgreicher Anstoß für erste Entwicklungen von vielversprechenden Versorgungsinnovationen zu sehen. Dieser Anstoß sollte aber nicht ab- sondern weiter ausgebaut werden. Wenn sie jetzt nach Meinung des Gesetzgebers (und geltendem Recht) mit dem 31.12.2008 auslaufen soll, müssten dafür andere Mittel und Wege gefunden werden, den gleichen oder einen noch besseren Effekt zu entwickeln. Höhere Qualität und mehr Wirtschaftlichkeit würden sich dabei nicht ausschließen, aber wie immer erfordert die Entwicklung von mehr Effizienz zunächst einmal das Investment in solche Effizienzsteigerungen und den Abbau entsprechender Hemmnisse. Hierfür marktorientierte Lösungen zu finden, halten wir für eine entscheidende, aktuell anstehende Aufgabe.

Die Innovation und Veränderung von Versorgungslösungen wird dabei immer auf die Widerstände derjenigen stoßen, die für sich in der Beibehaltung der aktuellen Versorgungslösung mehr Vorteile finden. Deshalb muss eine gesetzgeberische Initiative notwendigerweise Mittel und Wege suchen, wie die Innovatoren, wenn sie tatsächlichen Mehrnutzen für die Gesundheit der Bevölkerung entwickeln, belohnt werden.

Die Entwicklung von Versorgungsinnovationen ist im Übrigen nicht nur ein gesundheitspolitisches, sondern in vielfältiger Weise auch ein wirtschafts- und beschäfti- 


\section{THEMA}

gungspolitisches Thema. Hier können Erfahrungen aufgebaut werden, die der deutschen Gesundheitswirtschaft einen internationalen Wettbewerbsvorteil verschaffen können. Zahlreiche Länder arbeiten an Überlegungen zur günstigeren und besseren Organisation der Gesundheitsversorgung und suchen nach Lösungen, die Qualität und Wirtschaftlichkeit miteinander zusammen bringen. Die bisher im Kontext der Anschubfinanzierung der Integrierten Versorgung in Deutschland entwickelten Modelle einer transsektoralen und zum Teil auch alle Indikationen überspannenden Versorgungsinnovation können dabei durchaus eine Leitfunktion für die Zukunft übernehmen.

Letzteres insbesondere deswegen, weil die US-amerikanischen Managed Care Modelle - aus deren Erfahrungsschatz sich die deutschen Eigenentwicklungen zum Teil bedient haben - vor dem Hintergrund des amerikanischen Gesundheitsmarktes in vielen Ländern eine gewisse Abstoßungswirkung entfalten und die Kombination einer Integration der Versorgung mit einem solidarischen aber wettbewerblich organisierten Krankenkassensystem durchaus reizvoll erscheint. Eine kluge Entfaltung von Versorgungs- und Systeminnovationen kann deshalb für die deutsche Gesundheitswirtschaft und die Weiterentwicklung der gesetzlichen wie auch privaten Krankenversicherung ein ganz entscheidender Erfolgsfaktor werden. Dies gilt insbesondere für die angestrebte Angleichung der Gesundheitssysteme auf europäischer, aber auch auf einer übergeordneten globalen Ebene.

\section{Ausrichtung von F\&E für Versorgungsinnovationen im Gesundheitswesen}

Ende 2007 hat ein Expertengremium in einem MetaForum „Innovation im Gesundheitswesen“, veranstaltet von dem Fraunhofer Institut für System- und Innovationsforschung, eine exzellente Beschreibung von Innovationen im Gesundheitswesen entwickelt, dem wir uns hier anschließen wollen:

„Innovation im Gesundheitswesen ist nicht nur die Entwicklung und Einführung neuer Produkte, Prozesse und Strukturen. Vielmehr wird ein grundlegender Perspektivenwechsel erforderlich, um grundsätzlich neue Potenziale zur Verbesserung des Gesundheitssystems erkennen und realisieren zu können. Dies gilt für viele Bereiche, vor allem bedeutet es aber einen Paradigmenwechsel von einer krankheitsorientierten zu einer gesundheitserhaltenden Strategie, die Ausrichtung des Systems auf Bürger, Versicherte und Patienten als aktiv Handelnde, die ganzheitliche Berücksichtigung ihres individuellen Bedarfs, und die alle Politikfelder übergreifende Betrachtung der Gesundheit als entscheidende Grundlage und Chance. “9

Der etwas engere Bereich der Versorgungsinnovationen, das heißt das Aufbrechen der sektoralen Schranken (bzw. in der Wirtschaft würde man sagen, das „Reengineering“ der Versorgungsprozesse), muss die oben angesprochene Ausrichtung mit aufnehmen. Das heißt, es muss um die gezielte gesamtgesellschaftlich sinnvolle Entwicklung von
Gesundheitsnutzen stiftenden Versorgungsinnovationen gehen: Innovationen müssen sich ausrichten auf einen überlegenen Kosten-Nutzen des veränderten Prozessgeschehens gegenüber der herkömmlichen Vorgehensweise. Dieser Kosten-Nutzen wird bei bei den in ihrer Prävalenz besonders wichtigen kostenintensiven chronischen Krankheiten nicht von heute auf morgen nachzuweisen, sondern wird auf mehrere Jahre zu rechnen sein und durchaus komplexe Evaluationsformen benötigen. Wichtiger ist es, die Entwickler und ihre möglichen Vertragspartner bei den Krankenkassen dafür zu incentivieren, diese gesamtgesellschaftlich wichtige Aufgabe nicht erst morgen, sondern bereits heute in Angriff zu nehmen.

\section{Was hat bisher die Umsetzung von Versorgungsinnovationen behindert?}

An dieser Stelle wird im Gesundheitswesen immer schnell auf die Unbeweglichkeit der körperschaftlichen Strukturen verwiesen und den etablierten Machtzentren vorgeworfen, sie wollten diese nicht mit evtl. neu entstehenden Zentren teilen. Dieser Vorwurf ist im Großen und Ganzen sicherlich berechtigt, u. E. aber nicht die Hauptursache. Auch die Komplexität der emotionalen Seite des Geschehens, da Unverständnis und der Konflikt zwischen Niedergelassenen und dem Krankenhaus, die Angst vor dem Verlust der Selbständigkeit, die Sorge um das Transparentwerden von Qualitätsmängeln etc. lassen sich als Gründe argumentieren - und vielerorts als eine der Scheiternsursachen finden, erklären aber immer noch nicht hinreichend die Vorsicht, mit der das Thema bisher angegangen wird. Unseres Erachtens (und hier sind wir uns einig mit den Schlussfolgerungen eines 2003 in der Zeitschrift für Arbeit und Sozialpolitik erschienenen Artikels ${ }^{10}$ ) muss primär einer ökonomischen Analyse der Vorrang gegeben werden.

Entwickelt und investiert wird da, wo mit hinreichender Sicherheit nachhaltig mit unternehmerischem Engagement Geld verdient werden kann. Und genau diese hinreichende Sicherheit fehlt bisher. Im Grunde mangelt es einer unternehmerischen Herangehensweise und dies erklärt sich aus folgenden Faktoren:

Hohe Vorab-Investitionskosten: Die Unterschiedlichkeit der Systeme ambulant und stationär, Heilmittel, Pharma etc. erfordert eine aufwändige und komplexe Neuentwicklung z.B. von

- Ablaufstrukturen („integrierte Behandlungsleitfäden“),

- Synthese unterschiedlicher Codierungen und IT-Systeme (ICD 9,10 für Ärzte und Krankenhäuser unterscheiden sich, es gibt eine Vielzahl unterschiedlicher IT-Systeme und viele Ärzte arbeiten noch ganz ohne EDV) und

- Leistungsabrechnung (HVM/EBM im Vertragsärztebereich, Pflegesätze bzw. DRGs im KH-/Reha-Bereich, 
GOÄ im Privatbereich) bei Nebeneinander von herkömmlicher Finanzierung und IV-Finanzierung

- Unsicherheit der möglichen Refinanzierung der Investitionskosten:

- Unsicherheit der Investition: Werden Krankenkassen und Politik auf Dauer IV honorieren? Kann die Kostenentwicklung durch Morbiditätsveränderung und technolog. Entwicklung dauerhaft abgebildet werden? Wie verändern sich die Rahmenbedingungen aufgrund der Einführung des Gesundheitsfonds und des Morbi-RSA?

- Können die international gesehenen Einsparungspotenziale auch in Deutschland erreicht werden?

Gleichzeitig müssen die Unsicherheiten und Egoismen der jeweiligen Partner in diesem Veränderungsprozess berücksichtigt werden. Das beginnt bei den Kassen und geht über die Kassenärztlichen Vereinigungen, die Niedergelassenen, die Krankenhäuser bis hin zu den Patienten.

Eine erfolgreiche Weiterentwicklung der Integrierten Versorgung und die Schaffung einer grundsätzlichen Kultur sowie der entsprechend erforderlichen Etats für Forschung und Entwicklung (F\&E) für Versorgungsinnovationen müssen insofern auf verschiedenen Ebenen zugleich ansetzen.

\section{Vorschlag eines Maßnahmenpaketes "Forschung und Entwicklung (F\&E) für Versorgungsinnovationen" nach Ablauf der Anschubfinanzierung}

In seinen Vorschlägen lässt sich das White Paper von dem Gedanken leiten, dass die beste Förderung von „Forschung und Entwicklung (F\&E) für Versorgungsinnovationen " dann gelingt, wenn die Finanzierung implizit in den Marktregularien unseres zwar wettbewerblich angelegten aber nach wie vor körperschaftlich geregelten Gesundheitswesens enthalten ist. Jede separate Förderung auf Zeit hat dagegen immer auch problematische Anreizwirkungen, dennoch kann gelegentlich auf eine solche Anstoß gebende Wirkung nicht verzichtet werden. In dem White Paper werden deshalb Elemente aus den beiden Anreizwegen kombiniert.

\subsection{Kapitalfonds zur Investitionsfinanzierung für Unternehmensformen}

Wenn die von uns (und anderen) beschriebene Situation korrekt wieder gegeben ist, dann fehlt zur nachhaltigen Entwicklung von Versorgungsinnovationen eine entsprechend nachhaltige Verdienstmöglichkeit und eine ausreichende Kapitaldecke.

Als Lösung für letzteres wird im White Paper die Prüfung folgender Gestaltungsoptionen vorgeschlagen:
- Gründung eines zeitbegrenzt auf zehn Jahre angesetzten Kapitalfonds zur Investitionsfinanzierung für Unternehmensformen im Bereich von Versorgungsinnovationen entsprechend der in 1.4 beschriebenen Ausrichtung

Prüfung, ob ein solcher Kapitalfonds mit anteiligen Sicherheitsverzichten und begünstigten Zinssätzen besser als wirtschaftspolitische oder gesundheitspolitische Initiative umgesetzt werden sollte.

Für das Erstere - also die wirtschaftspolitische Initiative - wäre evtl. eine eigenständige Förderung über die Bankengruppe der Kreditanstalt für Wiederaufbau (analog ihrer Mittelstandsförderung) denkbar, für das Zweite - also die gesundheitspolitische Initiative - wäre an eine direkte entsprechende Förderung aus dem Gesundheitsfonds zu denken.

Beide Fondslösungen ließen sich Verknüpfungen mit evtl. spezieller zusätzlich begünstigter Förderung von Unternehmensgründungen, die auf spezifische gesundheitspolitisch gewünschte Ziele hin arbeiten, so z. B. für die Aufrechterhaltung von medizinischer Versorgung in ländlichen Räumen, die spezielle Beachtung der Einbeziehung von älteren Arbeitnehmern oder sozial schwächeren Bevölkerungsteilen oder auch Genderaspekten in die Versorgungsinnovation.

Insbesondere für die Gesundheitsfonds-Lösung würde sich eine begleitende Evaluationsverpflichtung anbieten, die Antragsteller auf eine unabhängige KostenNutzen-Analyse ihrer Ergebnisse über einen ausreichend langen Zeitraum hin verpflichtet und die dafür benötigten Mittel in einem ausreichenden Maße zur Verfügung stellt.

Zum speziellen Anreiz und zur Belohnung von Unternehmensgründungen mit positivem gesamtgesellschaftlichem Kosten-Nutzen-Verhältnis könnte ein teilweiser Rückzahlungsverzicht an das Outcome der Ergebnisevaluation gebunden werden.

\subsection{F\&E-Etat im Gesundheitsfonds sowie Gesundheitsnu tzensberichtsverpflichtung der Krankenkassen}

Mittel- bis langfristig wirkende Versorgungsinnovationen, insbesondere solche mit Einbeziehung von präventiv wirksamen Elementen, führen Krankenkassen und Versicherungen in die Problematik, dass die Kosten heute zu Buche schlagen, die Erträge aber erst zeitlich verschoben produziert werden. Zu diesem Zeitpunkt aber könnte a) der Versicherte die Krankenkasse bereits verlassen haben und/oder b) die Krankenkasse selber könnte bereits nicht mehr existieren, da sie aufgrund der erhöhten heutigen Kosten evtl. im Wettbewerb um Erstattungen bzw. Zusatzbeiträge nicht mehr bestehen konnte. Aus diesem Dilemma entsteht ein Disincentive für $\mathrm{F} \& \mathrm{E}$.

Eine besondere Chance besteht - und diese Position wird mit dem White Paper vertreten - in der Verpflichtung zur qualitäts- und ergebnisbezogenen Berichterstattung sowie 


\section{THEMA}

in der langfristigen Befreiung der Krankenkassen aus der Kameralistik und dem Rechtsrahmen der Körperschaft öffentlichen Rechts - insbesondere letzteres auch unter dem Gesichtspunkt der europaweiten Konvergenz und damit der Export- und Weiterentwicklungschancen der deutschen Krankenkassen und Versicherungsunternehmen. Der aktuell vorgelegte Kabinettsentwurf des GKV-Organisationsweiterentwicklungsgesetzes enthält hier durchaus erste Ansätze, etwa in der Annäherung an das HGB, müsste aber noch weiter daraufhin optimiert werden.

Als Lösung wird im White Paper die Prüfung folgender Gestaltungsoptionen vorgeschlagen:

In den Haushalts- und Buchführungsvorschriften der Krankenkassen wird eine eigene Regelung für die Behandlung von F\&E-Investitionen eingeführt. Der Spitzenverband Bund der Krankenkassen bereitet im Einvernehmen mit dem Bundesministerium für Gesundheit die Bedingungen vor, unter denen entsprechende sächliche wie Dienstleistungsinvestitionen als F\&E kategorisiert werden können und abschreibungsfähig (und auf welchen Zeitraum) wären, über das Bundesversicherungsamt würde die Kontrolle erfolgen.

- In Fortsetzung des Gedankens der 1\% Anschubfinanzierung wird ein z. B. auf 1- 3\%-erhöhter Anteil an den Gesamtbeitragseinnahmen des Gesundheitsfonds einem F\&E-Konto zugeführt. Über dieses F\&E-Konto kann die einzelne Krankenkasse ihrem ihr zustehenden Gesundheitsfondsanteil entsprechend verfügen, allerdings muss sie entsprechende F\&E-Investitionen im Nachgang nachweisen. Auch hier wiederum können die Anforderungen an erfolgreiche Versorgungs- und Systeminnovationen, wie sie in 1.4. definiert wurden, als Grundlage genommen werden. Wenn sie dazu nicht in der Lage ist, dann muss sie die Gelder an den Fonds zurückzahlen und diese werden dem Fondsvermögen für das nächste Jahr zugeführt.

Krankenkassen und Versicherungen sind gleichzeitig darauf zu verpflichten, innerhalb eines angemessenen Zeitraums Qualitäts- und Ergebnisberichte des Gesundheitszustands ihrer Versicherten und seiner Entwicklung über die Zeit - in Erweiterung der BQS-Berichterstattung für Krankenhäuser - und einen Bericht über ihre Investitionen in die F\&E für Versorgungsinnovationen zu veröffentlichen. Diese Berichte sollen den Versicherten und der Öffentlichkeit entsprechende Transparenz und Entscheidungshilfe geben und ermöglichen, diejenige Krankenkasse auszusuchen, die ihnen am ehesten durch gute selektiv- wie kollektivvertragliche Versorgungssysteme die Gewähr für einen hohen eigenen Gesundheitsnutzen bietet. Jenseits des Vergleichs der Zusatzbeiträge (und damit der Kosten) werden damit Krankenkassen belohnt, die sich durch erfolgreiche Anstrengungen in Primär- wie Sekundärpävention von den anderen abheben. Der Gemeinsame Bundesausschuß könnte hierfür in enger Zusammenarbeit mit den Vertretern der Patienten und Versicherten sowie der Bundesgeschäftsstelle Qualitäts- sicherung ein entsprechendes Anforderungsmodell an derartige Berichte entwickeln.

— Es ist zu befürchten, dass die Rechtsform der Körperschaften öffentlichen Rechts für ein aktives investives Verhalten der Krankenkassen mittelfristig nicht ausreichen könnte. Deshalb sollte der Gesetzgeber darüber hinaus prüfen, inwiefern auch Gesetzlichen Krankenkassen Rechtsformen jenseits der Körperschaften öffentlichen Rechts ermöglicht werden können, etwa im Sinne der Versicherungsvereine auf Gegenseitigkeit. In diesem Zusammenhang sind eine umfangreiche Prüfung der vergaberechtlichen und steuerrechtlichen Vorschriften sowie die Entwicklung eines Regelungsrahmens vorzusehen, um eine rechtliche Behinderung der neuen Rechtsformen von vornherein zu vermeiden, eine öffentliche Regulierung aber dennoch durchzusetzen. Vorbilder in anderen europäischen Ländern wie der Schweiz oder den Niederlanden existieren bereits.

\subsection{Pay-for-Performance Vergütungen für Leistungserbringer}

Die Leistungserbringer, ob aus der Ärzteschaft, den Krankenhäusern, der Pflege oder auch aus anderen Bereichen des Gesundheitswesens, sehen sich heute damit konfrontiert, dass ihre Leistung in ihrer Vergütung

unabhängig von dem damit geschaffenen Gesundheitsnutzen ist, d.h. dem gesellschaftlich geschaffenen Wert (die abrechenbare DRG eines Krankenhauses differiert so z.B. nicht nach der Mehr- oder Minderinanspruchnahme an vertragsärztlichen Leistungen im anschließenden Jahr aufgrund einer evtl. erhöhten Gesundheitskompetenz des Patienten, die ihm im Krankenhaus durch entsprechende Schulungen vermittelt worden wäre);

- unter dem Druck von bereits heute möglichen Ausschreibungen in Selektivverträgen steht. Leistungserbringer befürchten in diesem Zusammenhang, dass bei einer solchen Ausschreibung Krankenkassen dem kurzfristigen Anreiz des niedrigsten Preises auf Kosten des mittel- bzw. langfristig kostengünstigsten Preis-Leistungsverhältnisses nachgeben könnten.

Beide Situationen führen für die Leistungserbringer zu einem Disincentive für $\mathrm{F}+\mathrm{E}$ der von uns beschriebenen Ausrichtung. Das Interesse der Leistungserbringer ist dagegen einseitig auf eine Senkung von Produktionskosten und die Erhöhung der Leistungsmengen ausgerichtet. Während ersteres durchaus gesamtwirtschaftlich zu begrüßen ist, hat der zweite Anreiz im Gesundheitswesen mehrheitlich problematische Züge.

Als Lösung schlagen wir im White Paper die Prüfung folgender Gestaltungsoptionen vor:

Als Belohnung von höherer Outcome-Qualität gestützt durch Ergebnisdaten, die über die einzelnen Sektoren hinausgehen, schlagen wir im White Paper die gesetzgeberische Unterstützung und Ausrichtung der Kranken- 
kassen auf „Pay for Performance“ Vergütungen vor, wie sie in den USA und in UK für Krankenhäuser und z. T. Ärztegruppen eingeführt werden bzw. bereits eingeführt worden sind. Dabei gilt es zu beachten, dass keine steuerrechtlichen Hürden für die Leistungserbringer aufgebaut werden.

- Zur Vermeidung von „Gaming“-Effekten von Krankenkassen mit Leistungserbringern gegen die Versicherten sollte seitens des Gesetzgebers geprüft werden, alle selektivvertraglichen Regelungen auf die Einfuihrung von ergebnisabhängigen Verguitungsanteilen zu verpflichten. Wir sind uns bewusst, dass entsprechende Berechnungsmethoden erst über einen längeren Zeitraum bei entsprechender Datenqualität entwickelt werden können und dass die heutigen Datenschutzanforderungen zum Teil einer solchen transparenten Bewertung entgegenstehen. Eine Anpassung der Datenschutzanforderungen halten wir in der Abwägung zwischen dem Daten- und dem Gesundheitsschutz für notwendig.

\subsection{Shared Value-Modelle für die Industrie}

Die gesundheitsnahe Industrie reicht von Pharmaherstellern über Medizintechnik, Telemedizin und Medizinsoftware bis hin zu Biotech-Industrie, Disease Management Dienstleistern und den aufkommenden Telemonitoring-Dienstleistern. In gewissem Sinne lassen sich auch die Medizinischen Versorgungszentren und Managementgesellschaften der Integrierten Versorgung der gesundheitsnahen Industrie zuordnen, auch wenn diese systematisch den Leistungserbringern angehören. Sie stehen insbesondere vor dem Problem der Sektorengrenzen, d. h. dass ein Produkt oder eine Dienstleistung jenseits des Sektors Nutzen entfaltet, in dem es eingesetzt wird, dem anwendenden Leistungserbringer aber dieser Nutzen nicht zum Vorteil gereicht sondern sogar noch das Budget belastet. Auch hier findet sich ein Disincentive gegen die Weiterentwicklung von intelligenten Versorgungslösungen. Mit den Risk Share und Shared Value-Modellen der Verträge nach § 130 a SGB V sind hier erste übergreifende Lösungen entwickelt worden, derartige Vertragsformen gilt es weiter zu entwickeln.

Als Lösung schlagen wir im White Paper die Prüfung folgender Gestaltungsoptionen vor:

- Ausweitung der Vertragsmöglichkeiten zwischen Anbietern im Bereich der gesundheitsnahen Industrie sowie den Krankenkassen und Krankenversicherungen nach dem Modell des § 130 a im Sinne der Entwicklung von Versorgungsinnovationen

- Herausnahme von Risk Share und Shared ValueModellen aus der Jurisdiktion des Vergaberechts - auch schon im Vorwege einer Befreiung der Krankenkassen aus den Regelungen der Körperschaft öffentlichen Rechts.

\subsection{Ausweitung von Wahltarifen}

Die Vermehrung der Wahlfreiheiten der Versicherten hat sich bisher vor allem auf die Beitragssatzhöhe bezogen. Inzwischen ist es aber Zeit, hier durch entsprechende Transparenzvermehrung auch die qualitative und ergebnisbezogene Seite stärker zu betonen, zumal die Versicherten und Patienten die Hauptleidtragenden fehlender Prozessinnovationen im Gesundheitssystem sind. Die bei den Krankenkassen beschriebene Pflicht zu Qualitäts- und Ergebnisberichten würde hier schon einen ersten Anreiz setzen, die F+E Verpflichtungen und Befreiungen ebenso wie die anderen Vorschläge in den Teilabschnitten 1.4.1 - 1.4.4 werden ebenso Nutzen stiften, dennoch halten wir weitere Regelungen für notwendig.

Als Lösung schlagen wir im White Paper die Prüfung folgender zusätzlicher Gestaltungsoptionen vor:

- Ausweitung der Wahltarife für Krankenkassen mit der Möglichkeit, auch regionalisierte Wahltarife für kleinere Regionen anzubieten (da die Einführung von Wahltarifen heute mit relativ hohen Transaktionskosten verbunden ist, sollte hier durch den Gesetzgeber geprüft werden, ob die Anforderungen an derartig kleinräumig regionalisierte Tarife verringert werden könnten)

- Ausweitung der Möglichkeiten von präventiven Wahltarifen mit variabler Laufzeitfestlegung (im Gegenzug zur nachgewiesenen Mitarbeit der Versicherten an der Senkung ihres Morbiditätsrisikos und bei definierten Investitionsverpflichtungen der Krankenkassen in Prävention)

\section{Diskussion}

\subsection{Diskussion des Vorschlags für einen F\&E-Etat im Gesundheitsfonds}

Verständlicherweise konzentrierte sich die Diskussion des White Papers in der Fachöffentlichkeit auf den Vorschlag zur Einführung des F\&E-Etats im Gesundheitsfonds. Während der Vorschlag von Verbraucherseite und der Seite der Leistungserbringer eher positiv bewertet wurde, wurden aus dem Bereich der Gesundheitsökonomie und dem Bereich der Krankenkassen eher kritische Punkte bei meist grundsätzlicher Befürwortung des dahinter stehenden Anliegens vorgetragen:

- Der Vorschlag ist überflüssig, da der Morbiditäts-Risikostrukturausgleich schon selber wichtige Incentives setze, um die innovative Versorgung schwer Erkrankter zu befördern.

- Der Vorschlag entziehe dem Gesundheitsfonds Gelder, die aber dringend für die Versorgung der Versicherten gebraucht würden.

- Die Entwicklung einer Forschungs-, Entwicklungs- und Innovationspolitik sei eine gesamtgesellschaftliche Aufgabe und insofern könnten die Solidargemeinschaften diese Aufgabe nicht alleine schultern. 


\section{THEMA}

- Die Verwaltung des Fonds und Begutachtung der Richtigkeit der vorgelegten Abrechnungen gebäre eine neue Bürokratie und Planwirtschaft mit Fragen, etwa wer die Richtigkeit nach welchen Kriterien kontrolliere, und wie vermieden werden könne, dass dies als „Spielgeld“ betrachtet werden könne und zu leichtfertiger Ausgabe anreize.

Auch wenn es richtig ist, dass der Morbiditäts-Risikostrukturausgleich die innovative Versorgung schwer Erkrankter befördern kann, so enthält er doch das Problem der Kurzzeitigkeit seiner Anreize. Eine innovative Versorgung, die im ersten Jahr teurer und erst in den Folgejahren für die Krankenkasse zu einem Return-on-Investment führt, bleibt erschwert und wird in der Situation von Unsicherheit bzgl. der evtl. Erhebung von Zusatzbeiträgen noch zusätzlich erschwert. Es ist insofern nicht erstaunlich, dass viele Krankenkassenvertreter, so u.a. Christoph Straub, genau diese Gefahr massiv bedauern.

Nicht ganz folgerichtig ist der Einwand, dass dem Gesundheitsfonds mit diesem Vorschlag Geld entzogen würde, das die Krankenkassen für die Versorgung der Versicherten benötigten. Dadurch, dass den Krankenkassen vollständig der ihnen zustehende Anteil am Fonds zugewiesen wird und sie nur nachträglich die zweckgemäße Verwendung zugunsten von Versorgungsinnovationen nachweisen müssen, wird genau dieser Sorge Rechnung getragen. Zum einen sind die heutigen Ausgaben für IVVerträge schon Gegenstand der in den Fonds und dessen Beitragsfestsetzung einlaufenden heutigen Kosten, zum anderen sollen und werden mit diesen Mitteln ja durchaus Kosten von Krankheiten beglichen. Daneben wurden ordnungspolitische Bedenken gegen die Etatisierung und den damit verbundenen Eingriff in die Entscheidungsautonomie der Sozialleistungsträger geäußert. Dem läßt sich entgegen, dass der Vorteil der Etatisierung der Logik körperschaftlicher Verwaltung durchaus entspricht (und nach wie vor unterliegen die Krankenkassen dieser eher körperschaftlicher Logik): Die Festsetzung eines sektorenübergreifenden Budgets und einer Ausgabenverantwortlichkeit verlangt auf der inneren Ebene der Krankenkasse eine entsprechende sektorenübergreifende Verwaltung. Damit kann eine Gesamtverantwortung gestärkt werden, die ansonsten der sektoralen Aufgliederung der inneren Organisation der Krankenkasse zum Opfer fallen könnte. Und solange keine Abschreibungsfähigkeit von Investitionen ermöglicht wird, kann in diesem Zuge und mit diesem Modell auch eine Investition mit einer Refinanzierungsfrist von mehr als einem Jahr für die Krankenkasse angereizt werden.

Die gesamtgesellschaftliche Aufgabe zur Entwicklung einer Forschungs-, Entwicklungs- und Innovationspolitik drückt sich nach Ansicht des Verfassers u.a. in den - sich im Laufe der nächsten Jahre erhöhenden - Steuerbeiträgen zum Gesundheitsfonds aus. Außerdem würde dies noch durch den gleichfalls gemachten Vorschlag eines Kapitalfonds ergänzt, der etwa durch die KfW-Bankengruppe verwaltet werden könnte. Vom Grundsatz her sollte allerdings nachdrücklich die Entwicklung einer eigenen F\&E-Kultur in den Krankenkassen und bei den Leistungserbringern gefördert und gefordert werden. Langfristig kommen wir nicht umhin, dass diese Branche, wie jede andere Wirtschaftsbranche auch, selber für die eigene Erneuerung ihrer Abläufe und Versorgungsprozesse sorgt. Bisher bestimmt das immer noch vorhandene Denkmuster der Kostenerstattung viel zu stark die eigene Budgetplanung, und das kurioserweise obwohl die Kostenerstattungspolitik inzwischen schon über eineinhalb Jahrzehnte abgelöst wurde.

Die sicherlich schwierigste Frage ist die der Verwaltung und Regelung des F\&E-Etats ohne Heranzüchtung neuer Bürokratien. Nach Ansicht des Verfassers könnte hier ein vereinfachtes Verfahren der Nachweisdarlegung und Stichprobenprüfung eingeführt werden. Für letzteres würde sich wahrscheinlich eher das BVA als der Spitzenverband Bund eignen. $\mathrm{Zu}$ diskutieren wären hier gewisse Berichts- und Beforschungspflichten über die gesundheitsbezogenen Outcomes und die Erträge der Investitionen im Zeitablauf. Eine Karenzzeit von 3-5 Jahren sollte dabei den einzelunternehmerischen Vorteil erhalten und gleichzeitig die gesamtgesellschaftliche Weiterentwicklung ermöglichen. Als Kriterien für Versorgungsinnovationen im Sinne des F\&E-Etats bieten sich nach Ansicht des Verfassers folgende Bestandteile an:

Sie gehen direkt an die tatsächliche Veränderung und Optimierung der Ablaufprozesse heran;

Sie entwickeln eine umfassende Planung der gesamten Behandlungskette;

- Sie entfalten wirksamen Anreize für Prävention;

- Sie achten zwar die jeweiligen wirtschaftlichen Interessen der einzelnen Leistungserbringer, bringen diese aber in ein wirtschaftliches Anreizsystem, das die Qualität des Ergebnisses der Arbeit belohnt und nicht die Anzahl der Leistungen;

- Sie übernehmen einen Anteile an Gesamtverantwortung gegenüber den Krankenkassen und dem Patienten;

- Sie beziehen den Patienten aktiv in die Planung und Absprache der Therapie (als „Co-Therapeut“) ein und etablieren eine formalisierte aktive Patientenbeteiligung;

- Sie unterziehen ihre Arbeit und deren Ergebnisse einer intensiven Evaluation und kooperieren eng mit der Versorgungsforschung.

Die Gefahr der Anreizung einer Spielgeld-Haltung mit der Gefahr zu leichtfertiger Ausgaben sieht der Verfasser dagegen nicht. Die Drohung der Zusatzbeitragserhebung wird die Vorstände der Krankenkassen dazu zwingen, jede Ausgabe kritisch auf ihre Effekte gegenzuprüfen und nur Ausgaben zuzulassen, die eine hinreichende Gewähr auf Einsparung gegenüber den Normalkosten bieten. 


\subsection{Diskussion des Vorschlags zur Gesundheitsnutzens- berichtspflicht für Krankenkassen}

Dieser Vorschlag wurde zumeist eher positiv beurteilt, allerdings wurden einige Fragen dazu vorgetragen:

- Wie kann vermieden werden, dass hieraus nur neue Papierberge mit glatten Marketingbroschüren entstehen?

- Wie lange wird es dauern, bis die entsprechenden Daten für eine solche Nutzenstransparenz zur Verfügung stehen?

Zunächst soll mit diesem Vorschlag eine Orientierung gegeben werden: Krankenkassen sollen von den Versicherten nicht nur nach dem Gesichtspunkt der Beitrags- bzw. Zusatzbeitragssätze ausgewählt werden können und auch nicht nur nach dem heutigen Angebot von Wahltarifen etc. sondern auch nach der Betrachtung des langfristig erzeugten Gesundheitsnutzens für die Mitgliedschaft. Dem Verfasser ist dabei klar, dass ein fertiges Indikatorenset und ein Messverfahren noch nicht existiert, allerdings sieht er durchaus eine Reihe von Ansätzen, an die angeknüpft werden kann.

So eignen sich die GKV-Routinedaten, erst recht mit einer Adjustierung auf den Morbi-RSA, durchaus zu einer Analyse von Krankheitshäufigkeiten über den Verlauf der Zeit. Im Ausgleich zu dem tendenziellen Upcoding-Anreiz des Morbi-RSA würden bei der Nutzenberichterstattung Krankenkassen einen Vorteil im internen Krankenkassenwettbewerb um die Mitglieder erfahren, wenn sie erfolgreich Krankheiten bzw. Krankheitsprogressionen verhindern würden. Des weiteren kann an dem QSR-Pilotprojekt von AOK und Helios angesetzt werden, auch das sog. QUISA-Projekt für Qualitätsindikatoren in der vertragsärztlichen Versorgung und die Berichterstattung im Rahmen der BQS-Auswertungen können herangezogen werden.

Hervorgehoben sei hier noch einmal, dass dem Verfasser bewusst ist, dass eine derartige Berichterstattung nicht von heute auf morgen entwickelt werden kann allerdings eine Richtungsvorgabe - wie auch in der Qualitätsberichtspflicht für Leistungserbringer - durch den Gesetzgeber hilfreich sein kann. Selbstverständlich ist jeder Krankenkasse (und übrigens würde dies natürlich auch für die privaten Versicherungen gelten können) unbenommen, schon heute aus den vorhandenen Daten, erste Anfänge dazu zu erstellen und sich damit als Qualitätsanbieter vom Wettbewerb abzusondern (vgl. zum Beispiel die Berichte der mit Kaiser Permanente verkoppelten Organisation Group Health Cooperative of Puget Sound in der Region Seattle $\left.{ }^{11}\right)$.

\subsection{Diskussion des Vorschlags zur Verpflichtung auf Pay- for-Performance Vergütungen}

Auch dieser Ansatz wurde grundsätzlich eher positiv beurteilt, allerdings wurden Einschränkungen dazu vorgeschlagen:
So wurde eine Trennung in Kollektiv- und Selektivverträge empfohlen. Bei letzteren sollte die Ausweitung so weit wie möglich dem Wettbewerb überlassen werden. Wenn die Vertragspartner es als sinnvoll ansehen, hierbei Elemente von Pay-for-Performance vorzusehen, werden sie dies tun. Im nicht-wettbewerblichen Bereich der Kollektivverträge erscheine es eher vertretbar, administrative Zielvorgaben dieser Art zu machen.

Dieser Einschränkung schließt sich der Verfasser an.

\section{Schlussbetrachtung}

Die einfachen Kostensenkungen sind weitgehend ausgereizt, die „Zitrone“ ist bis auf wenige Bereiche ausgequetscht. Jetzt ist ein komplexeres „Reengineering“ der Versorgungskette gefragt, intersektorale Versorgungsund Systeminnovationen sind angesagt. Damit kommen wir aber schnell an die Frage der Refinanzierung solcher komplexer Architekturaufgaben. Das Bundesministerium für Gesundheit hat die handelnden Akteure um Vorschläge gebeten, wie bei Kostenträgern und Leistungserbringern eine Forschungs- und Entwicklungskultur für Versorgungsund Systeminnovationen im Gesundheitswesen aufgebaut und nachhaltig implementiert werden kann.

Dieser Aufforderung ist die Hildebrandt GesundheitsConsult GmbH nachgekommen und hat im Laufe des ersten Quartals 2008 Meinungsbildner aus allen Bereichen des Gesundheitswesens (Vertragsärzte, Krankenkassen, Krankenhäuser, Wissenschaft, Industrie) befragt. Aus den Antworten zu den Themen Anschubfinanzierung, den heutigen Hemmnissen für Forschung und Entwicklung ( $\mathrm{F}$ $\&$ E) und zur Einflussnahme durch den Gesetzgeber wurde der Vorschlag für die Entfaltung einer F \& E-Kultur für Versorgungs- und Systeminnovationen im Gesundheitswesen als White Paper entwickelt. In einer zweiten Diskussionsschleife wurden die Vorschläge den 190 Teilnehmern der Umfrage noch einmal vorgelegt, zur Diskussion gestellt und dann im Diskurs mit einigen der Teilnehmer auf einer Pressekonferenz am 3. Juni 2008 veröffentlicht.

Der Verfasser ist sich der Brisanz der Vorschläge und auch der berechtigten Kritik an einigen der Überlegungen durchaus bewusst, sieht andererseits einen dringenden Bedarf für eine offene weitergehende Diskussion und einen Mangel an alternativen Vorschlägen. Weitere Anregungen und eine umfangreiche Diskussion sind deshalb herzlich willkommen.

\section{Fußnoten:}

1 Quelle vgl.: http://www.bqs-register140d.de/dokumente/20080331.pdf 2 Vgl. das White Paper und den Videostream der Pressekonferenz unter: http://www.gesundheitsconsult.de/gc/archiv_-_news_und_updates/archiv_aktuelles_news_und_updates/080603whitepaper-final.html

3 Krauth, Ch., Schwartz, F. W., Perleth, M., Graf von Schulenburg, J.-M. : „Zur Weiterentwicklung des Vergütungssystems in der ambulanten ärztlichen Versorgung. Gutachten im Auftrag der Bundestagsfraktion Bündnis 90/Die Grünen“, 1997

4 Porter, M.E./Teisberg, E.O. (2006): Redefining Health Care - Creating Value-Based Competition on Results, Boston (Mass.) 


\title{
THEMA
}

5 Enthoven, A.C./Tollen, L.A. (2005): Competition in Health Care: It Takes Systems to Pursue Quality and Efficiency, in: Health Affairs, W5, p.420ff (7.Sept.2005)

6 Lüngen, M./Gerber, A./Lauterbach, K.W. (2008): Pay-for-Performance: Neue Impulse für den Wettbewerb zwischen Krankenhäusern, in: Klauber, J./Robra, B.-P./Schnellschmidt, H. (Hrsg.): Krankenhausreport 2007: Krankenhausvergütung - Das Ende der Konvergenzphase?, Stuttgart, New York

7 U.a. Hans-Jürgen Beckmann, Wilfried Boroch, Thorsten Bröske, Stefan Etgeton, Heinz Giesen, Gerd Glaeske, Robert Paquet, Rolf Stuppard, Veit Wambach, Jürgen Wasem, .... Vgl. http://www.gesundheitsconsult. de/downloads/pressemappe_mit_stellungnahmen_dritter.pdf
8 Stellungnahme zum Entwurf eines Gesetzes zur Modernisierung des Gesundheitssystems - GMG ..., Hildebrandt, H., vorgelegt zur Anhörung des Ausschusses für Gesundheit und Soziale Sicherung vom 23.6.2003 zur BT-Drs 15/1170 sowie 1174 und 1175

9 Memorandum zu Dimensionen der Innovation im Gesundheitswesen, MetaForum „Innovation im Gesundheitswesen“, Fraunhofer Institut für System- und Innovationsforschung, 10.Dez.2007, S.2.

10 Jacobs, Klaus (2003): Höchste Zeit für sinnvollen Wettbewerb in der GKV. In: Gesundheits- und Sozialpolitik Heft 3/4, März/April 2003, 57. Jahrgang. Baden-Baden: Nomos: 14-17

11 http://www.ghc.org/about_gh/co-op_overview/quality/ GroupHealthQualityPlan2008.pdf

\section{Festschrift für Norbert Klusen}

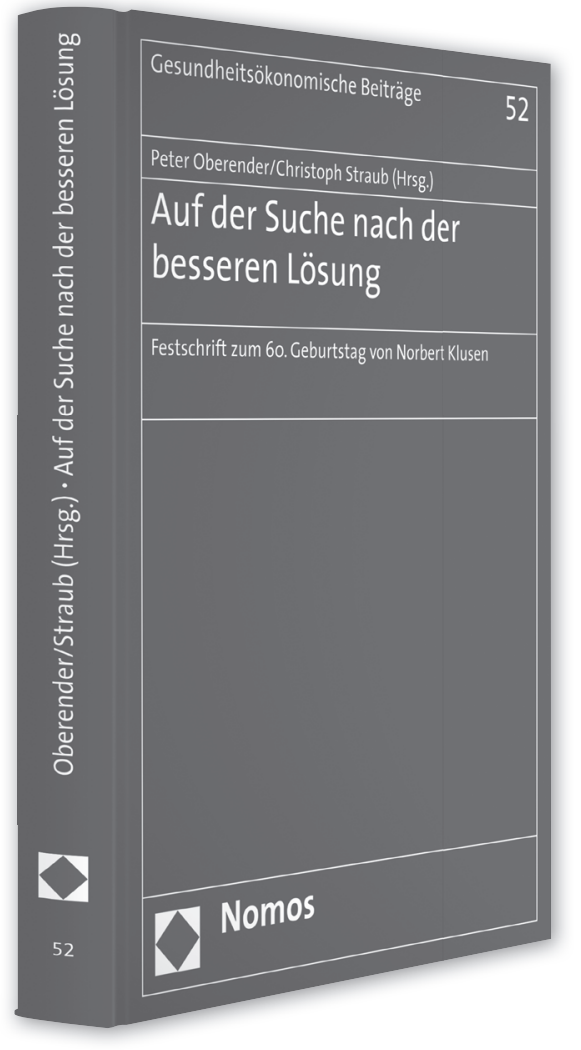

\author{
Auf der Suche nach der besseren Lösung \\ Festschrift zum 6o. Geburtstag von Norbert Klusen \\ Herausgegeben von Prof. Dr. Peter Oberender und \\ Dr. Christoph Straub \\ 2008, 267 S., geb., 49,- €, ISBN 978-3-8329-3111-7
}

(Gesundheitsökonomische Beiträge, Bd. 52)

Die Suche nach der besseren Lösung - das ist das Anliegen der 23 in diesem Band vertretenen Autoren. Repräsentanten aus Politik, Wissenschaft, Gesundheitswirtschaft und der gesetzlichen wie auch der privaten Krankenversicherung zeigen, wie sich unser Gemeinwesen und besonders das Gesundheitssystem verbessern ließen. Dazu werden Fragen der Gesundheitssystemforschung ebenso erörtert wie aktuelle Aspekte des Wettbewerbs zwischen gesetzlichen Krankenkassen.

Das deutsche Gesundheitssystem wird aus europäischer wie US-amerikanischer Sicht untersucht, der Gesundheitsbegriff aus ärztlicher Sicht thematisiert und Integrationsverträge rechtlich sowie hinsichtlich ihres Nutzens bewertet. 